The second patient is a 49 -year-old male who suffered at least 3 concussive blasts in the Army and a parachute injury. Following the last accident, the patient was diagnosed with major depressive disorder, panic disorder, PTSD and generalized anxiety disorder. He denies any psychiatric history prior to TBI including negative family history of psychiatric illness. In addition, he now suffers from nervousness, irritability, anger, emotional lability and concurrent concentration issues, problems completing tasks and alterations in memory.

Both patients underwent 1.5T multiparametric MRI using standard T2, FLAIR, DWI and T1 sequences, and specialized sequences including susceptibility weighted (SWAN/SWI), 3D FLAIR, single voxel MRI spectroscopy (MRS), diffusion tensor imaging (DTI), arterial spin labeling perfusion (ASL) and volumetric MRI (NeuroQuant). Importantly, this exam can be performed in 30-45 minutes and requires no injections other than gadolinium in some patients. We will discuss the insights derived from the MRI which detail the injured areas, validate the severity of the brain damage, and provide insight into the psychological, motivational and physical disabilities that afflict these patients. It is our expectation that this kind of imaging study will grow in value as we link specific patterns of injury to specific symptoms and syndromes resulting in more targeted therapies in the future.

\section{A Multiparametric MRI Protocol for Evaluation of Cognitive Insufficiency, Dementia and Traumatic Brain Injury (TBI): A Case Series}

John L. Sherman, $\mathrm{MD}^{1}$, Christen F. Kutz, PhD, $\mathrm{PA}^{2}$, Mitchell S. Szymczak, MS3 ${ }^{4}$, Deborah York, NP, PMHNP-BC, APRN, CCRA ${ }^{3}$ and Laurence J. Adams, MD ${ }^{2}$

${ }^{1}$ Colorado Springs Imaging, Colorado Springs, CO, USA, ${ }^{2}$ Colorado Springs Neurological Associates, Colorado Springs, CO, USA, ${ }^{3}$ Founder, Mountain Mind, LLC, Colorado Springs, CO, USA, and ${ }^{4}$ Western University of Health Sciences, Pomona, CA, USA

Presenting Author: John L. Sherman of dementia and TBI. As patients with these diseases are increasingly presenting to clinical practice, our ability to combine multiple parameters within the standard 30-minute or 45-minute (pre- and post-contrast) MRI exams has high potential to affect current and future clinical practice.

Methods. All MRI studies were performed on 1.5 T MRI GE 450w or GE HDx imagers. All patients were seen clinically in outpatient practices. All techniques are FDA approved. The 30 minute protocol utilized T2w FSE $3 \mathrm{~mm}, 2.5 \mathrm{~mm}$ SWAN, 3D T1 sagittal $1.2 \mathrm{~mm}$, DWI $5 \mathrm{~mm}, 3 \mathrm{D}$ FLAIR $1.2 \mathrm{~mm}, 2.5 \mathrm{~mm}$ SWAN (susceptibility sensitive), 3D T1 sagittal $1.2 \mathrm{~mm}$, arterial spin labeling perfusion, posterior cingulate single voxel PRESS MR spectroscopy and NeuroQuant automated volumetric analysis and LesionQuant automated lesion detection and measurement. The 45-minute TBI protocol added diffusion tensor imaging, MR spectroscopy (MRS) of normal appearing frontal white matter and 3D gadolinium enhanced technique.

Results. The combination of multiparametric data together with standard imaging and clinical information allowed radiologic interpretation that was able to focus on 1-2 specific diagnoses and to indicate those patients in which a combination of pathologies was most likely. Neurologists, gerontologists, neuropsychologists and psychiatric specialists used these data and our summary conclusions to develop more specific diagnoses, treatments and prognoses.

Conclusions. Readily available MRI techniques can be added to standard imaging to markedly improve the usefulness of the radiologic opinion in cases of subjective cognitive insufficiency, clinical mild cognitive insufficiency, behavioral pathologies, dementia and post-traumatic brain syndromes.

\section{Use of a Consultation Service Following Pharmacogenomic Testing in Psychiatry}

\author{
Daniel Dowd, PharmD and David S. Krause, MD
}

Genomind, Inc., King of Prussia, PA, USA

Presenting Author: Daniel Dowd

\section{Abstract}

Background. There is a plethora of drugs available to psychiatrists for treatment of mental illness, which can vary in efficacy, tolerability, metabolic pathways and drug-drug interactions. Psychotropics are the second most commonly listed therapeutic class mentioned in the FDA's Table of Pharmacogenomic Biomarkers in Drug Labeling. Pharmacogenomic (PGx) assays are increasingly used in psychiatry to help select safe and appropriate medication for a variety of mental illnesses. Our commercial laboratory offers PGx expert consultations by PharmDs and PhDs to clinician-users. Our database contains valuable information regarding the treatment of a diverse and challenging population. Methods. Genomind offers a PGx assay currently measuring variants of 24 genes relevant for selection of drugs with a mental illness indication. Since 2012 we have analyzed > 250,000 DNA 